\title{
Performance Evaluation of Novel MAC Protocol for WDM/Ethernet-PON
}

\author{
Bokrae Jung ${ }^{1}$, Hyunho Yun ${ }^{2}$, Jaegwan $\mathrm{Kim}^{1}$, Mingon Kim ${ }^{1}$, and Minho Kang ${ }^{1}$ \\ ${ }^{1}$ Information and Communications University (ICU), \\ 119 Munjiro, Yuseong-Gu, Daejeon 305-732, Republic of Korea \\ \{gaole3, tecmania, kmg0803, mhkang\}@icu.ac.kr \\ ${ }^{2}$ Electronics and Telecommunications Research Institute (ETRI), \\ 161 Gajeong-Dong, Yuseong-Gu, Daejeon 308-350, Republic of Korea \\ yhh63129@etri.re.kr
}

\begin{abstract}
This paper proposes a novel MAC protocol adopting time division duplex (TDD) technology for extending total subscribers at the expense of downstream bandwidth in WDM/Ethernet passive optical network (WE-PON). To compensate sacrificed downstream bandwidth, we employ an efficient threshold decision and dynamic bandwidth (DBA) algorithm. Simulation results show that the proposed MAC protocol improves queueing delay, link utilization under asynchronous traffic conditions.
\end{abstract}

\section{Introduction}

WDM-PON provides unlimited capacity with the each user enough to accommodate broadband multimedia services. Although WDM-PON is high-performance, it is difficult to be fully accepted today due to its luxurious cost. Therefore, WDM/TDM hybrid PON is a compromising solution guaranteeing cost-effective and flexible upgrade from TDM to WDM [1]. This paper introduces WE-PON architecture as a kind of WDM/TDM hybrid PON. In the WE-PON, single light source is reused at optical network termination (ONT) to modulate upstream signals called loop-back method for the efficient bidirectional transmission. Once adopting such a technology, splitting ratio is considerably limited due to amplitude squeezing effect (ASE) at ONT [2]. To address this problem, we propose an improved MAC protocol applying time division TDD technology [3]. Consequently, maximum 16 subscribers per wavelength can be serviced in the optical layer. But downstream bandwidth is inevitably taken away for upstream traffic in the network layer. To compensate downstream bandwidth used for up-traffic, threshold decision and dynamic bandwidth allocation (DBA) algorithm efficiently response to user request in order to keep tack with almost close performance of ASE-based WE-PON that does not use a proposed method.

\section{WE-PON Architecture}

Fig. 1 shows the overall architecture of WE-PON network. In the OLT, downstream signals from OLT multiplex 32 wavelengths into single mode fiber (SMF) through 
arrayed-waveguide gratings (AWGs). In the RN, downstream signals leaved from $10 \mathrm{~km}$ feeder link pass out 1 and port 2 of circulator for the right direction and then demultiplex 32 wavelengths for distributing signal power to 4 ONTs via optical power splitter (OPS). In the ONT, 25 percents of optical power splitted by OPS are fed into receiver. Remaining 75 percents are reused to modulate upstream signals using ASE in RSOA. By using ASE mechanism to regenerate continuous wave $(\mathrm{CW})$ to be modulated for upstream traffic in reflective semiconductor optical amplifiers (RSOA), strictly bounded power range of input signals should be allowed to enter the RSOA. It limits splitting ratio to only four. To solve this problem, we adopt TDD technology to the existing MAC protocol. As this scheme applies to existing frame structure, no more reuse of modulated downstream signals using ASE is need as well as maximum 16 subscribers per wavelength can be achieved.

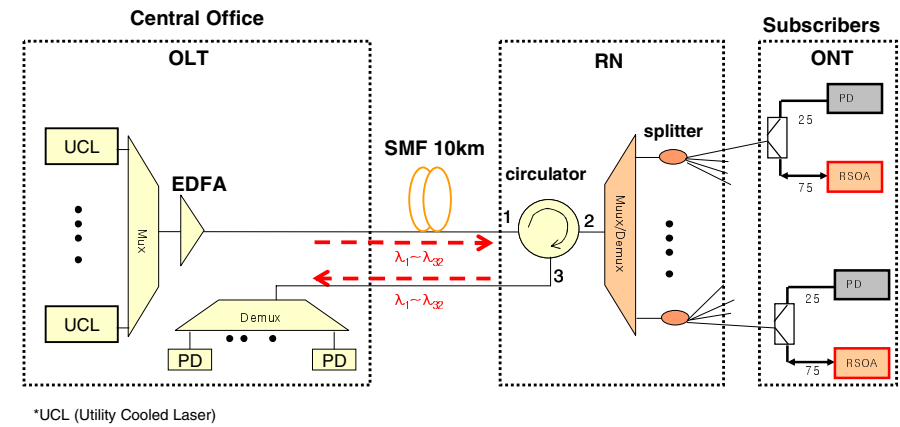

Fig. 1. WE-PON architecture

\section{Proposed MAC Protocol}

The basic downstream frame structure adopting TDD technology is shown in Fig. 2 . In this scheme, a part of the downstream area is shared with upstream bandwidth granted for ONTs. Here the threshold is regarded as the amount of upstream bandwidth. In this case, the length of upstream and downstream is evenly assigned, which call fixed threshold adjustment (FTA). In general, we design all the control message formats and collision avoidance mechanism among ONTs observe 802.3ah EFM. Within the threshold, a GATE message corresponding to the ONT's request, vacant time window for the $\mathrm{CW}$ amount to the grant size of the ONT and guard time are consecutively flow as a group to the ONT request. Contrast to FTA, the range of threshold can be adaptively varied from 0 to $2 \mathrm{~ms}$ according to upstream request for ONTs, called adaptive threshold adjustment (ATA) shown in fig. 3 .

Once adopting given MAC protocol in the scheduler, we can not avoiding degrading performance. But well designed threshold decision and DBA algorithm can im- 
prove system efficiency and capacity. Scheduling algorithm for determining the threshold and a mount of grant under the DBA policy is shown in fig. 4.

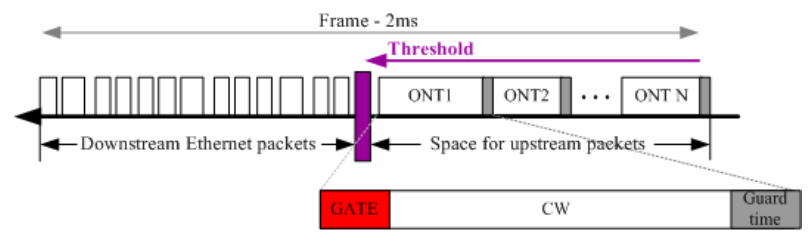

Fig. 2. Fundamental downstream frame structure

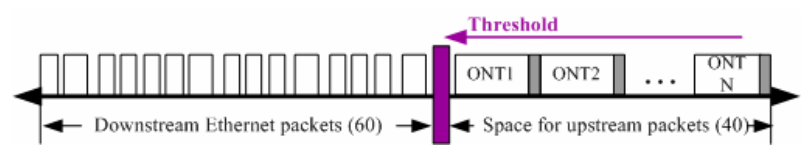

Fig. 3. Elastic threshold shift for ATA

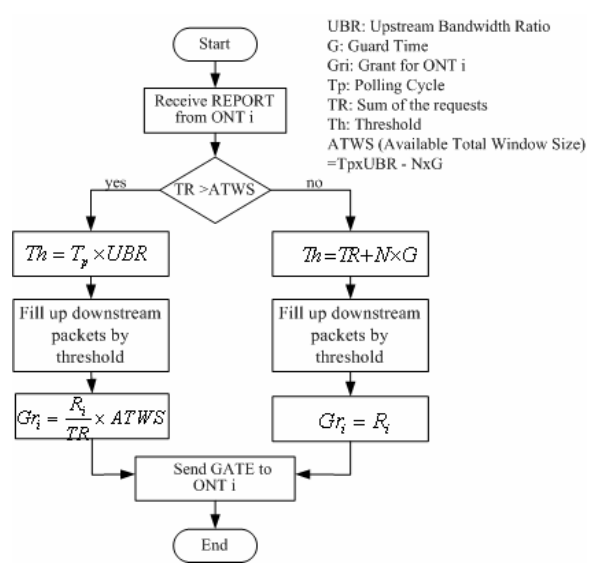

Fig. 4. DBA Algorithm for ATA

\section{Simulation Results}

We simulate the performance of proposed MAC protocol using OPNET. We assume distance from OLT to ONTs is identically $10 \mathrm{~km}$. Link speed for downstream and upstream transmission is set to $1 \mathrm{Gbps}$. Guard time between adjacent frames is let to 5us. Every ONT has a finite queue size, 20 Mbytes. Incoming Ethernet frame uniformly distributed from 64 to 1518 byte. Fig.5 shows average queueing delay, up-link utilization, and traffic scenario-based bandwidth efficiency for proposed MAC protocol. 


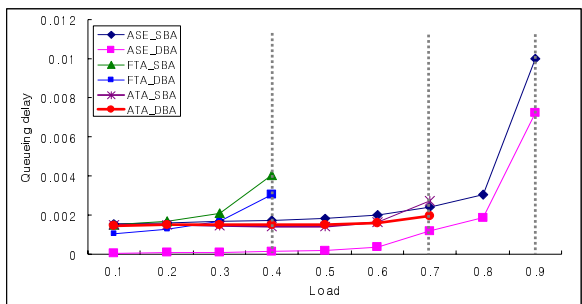

(a) Queueing delay

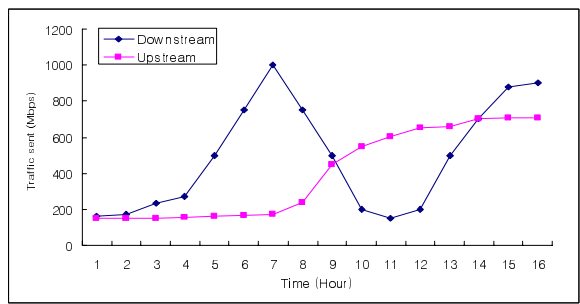

(a) Traffic Scenario

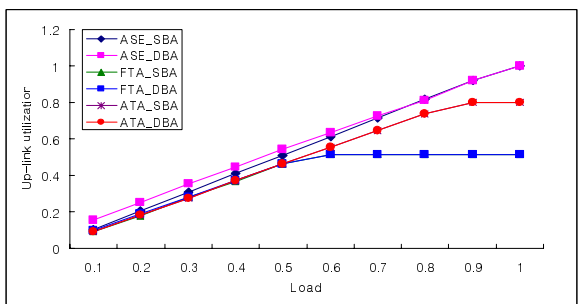

(b) Up-link utilization

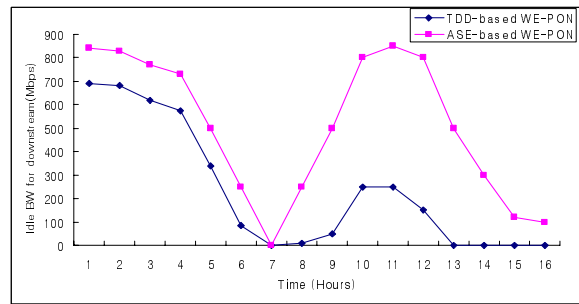

(b) Unused downstream bandwidth

Fig. 5. Performance evaluation for proposed MAC protocol

\section{Conclusion}

This paper proposed new MAC protocol adopting TDD technology to improve system efficiency of WE-PON. From the simulation results, the proposed MAC protocol achieved substantial improvement in queueing delay and link utilization under asynchronous traffic conditions by applying threshold decision and DBA algorithm for ATA.

\section{Acknowledgement}

This paper was supported in part by KOSEF-OIRC project and ETRI.

\section{References}

1. Fu-Tai An and Kyeong Soo Kim, "SUCCESS: a next-generation hybrid WDM/TDM optical access network architecture", J.Lightwave Technol., vol 22, no. 11, pp 2557 - 2569, Nov. 2004.

2. Wooram Lee and Mahn Yong Park, "Bidirectional WDM-PON based on gain-saturated reflective semiconductor optical amplifiers" IEEE Photon. Technol. Lett., vol. 11, no. 11, pp 2460 - 2462, Nov. 2005.

3. Huang, V. and Weihua Zhuang, "Optimal resource management in packet-switching TDD CDMA systems”, IEEE Personal Commun., vol. 7, no. 6, pp 26 - 31, Dec. 2000. 